\title{
Periodic $\mathrm{ZnO}$ nanorod arrays defined by polystyrene microsphere self-assembled monolayer
}

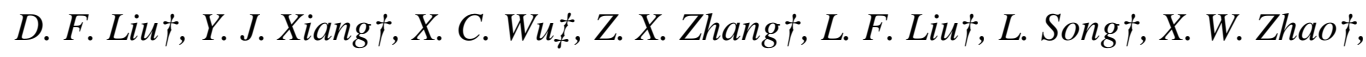

S. D. Luo†, W. J. Ma†, J. Shen†, W. Y. Zhou†, G. Wang †, C. Y. Wang†, S. S. Xie*†

$\dagger$ Beijing National Laboratory for Condensed Matter Physics, Institute of Physics, Graduate School of the Chinese Academy of Sciences, Beijing 100080, P. R. China

$\ddagger$ National Center for Nanoscience and Nanotechnology, Beijing 100080, P. R. China

*Corresponding author. E-mail: $\underline{\text { ssxie @ aphy.iphy.ac.cn }}$ 
Figure S1 shows the process of reduction of the size of PS microsphere using oxygen plasma etching. It indicates that the etching is isotropic and the spherical shapes are remained. The hexagonal periodicity is well preserved, although the sizes of the PS microspheres become smaller with the duration of oxygen plasma etching, which are very important for fabrication of well-patterned Au particles with tunable sizes in the next step of selectively etching Au with $\mathrm{Fe}^{3+} /$ thiourea aqueous solution.

Figure S2 presents the SEM images of the Au particle patterns obtained after the samples in Figure S1(b)-S1(d) were removed of the PS microspheres by ultrasonication in ethanol and selectively etched with the aqueous $\mathrm{Fe}^{3+} /$ thiourea solution. The size of the Au particles is $450 \mathrm{~nm}$ down to $250 \mathrm{~nm}$ for $4 \mathrm{~min}$ to $8 \mathrm{~min}$ oxygen plasma etching, indicating that it can be effectively tuned by the duration of the oxygen plasma etching. If the time of oxygen plasma etching is longer, or that of heating on the hot plate is shorter, or the size of the original PS microsphere is smaller, the size of the obtained Au particles will be smaller. And it's also revealed that the periodicity of the obtained Au particle arrays is completely reproduced from the PS microsphere self-assembly monolayer, that is to say, the quality of patterning of the $\mathrm{Au}$ particles is only depended on the original PS microsphere self-assembly monolayer.

Figure S3 shows the morphology change of Au particle patterns before and after annealing at $910 \square$. The thickness of ion-sputtered Au is about $1.5 \mathrm{~nm}$. The samples were sealed in a vacuumed quartz tube and delivered into the furnace tube when the furnace is at the desired temperature, and were cooled in the room temperature environment after annealing for $10 \mathrm{~min}$. It can be found that the particles with the 
diameter of $300 \mathrm{~nm}$ was turn into at least two tiny particles after annealing, those with the diameter of $200 \mathrm{~nm}$ all became one tiny particle after annealing but the periodicity of the pattern was damaged, however, after annealing, those with the diameter of 150 $\mathrm{nm}$ not only were turned into just one tiny particle but also made the hexagonal periodicity preserved. Therefore, it can be confirmed that more than one $\mathrm{ZnO}$ rods will be grown out on a single catalyzing site when the size of the used Au particle is larger than $200 \mathrm{~nm}$ due to its splitting into several tiny particles at the growth temperature; but when the diameter of the Au particle is about $150 \mathrm{~nm}$ or less, it shrinks into one tiny particle at the growth temperature while the order of the pattern is well preserved, so highly-ordered individually patterned $\mathrm{ZnO}$ rod arrays can be grown using this kind of $\mathrm{Au}$ particle patterns.
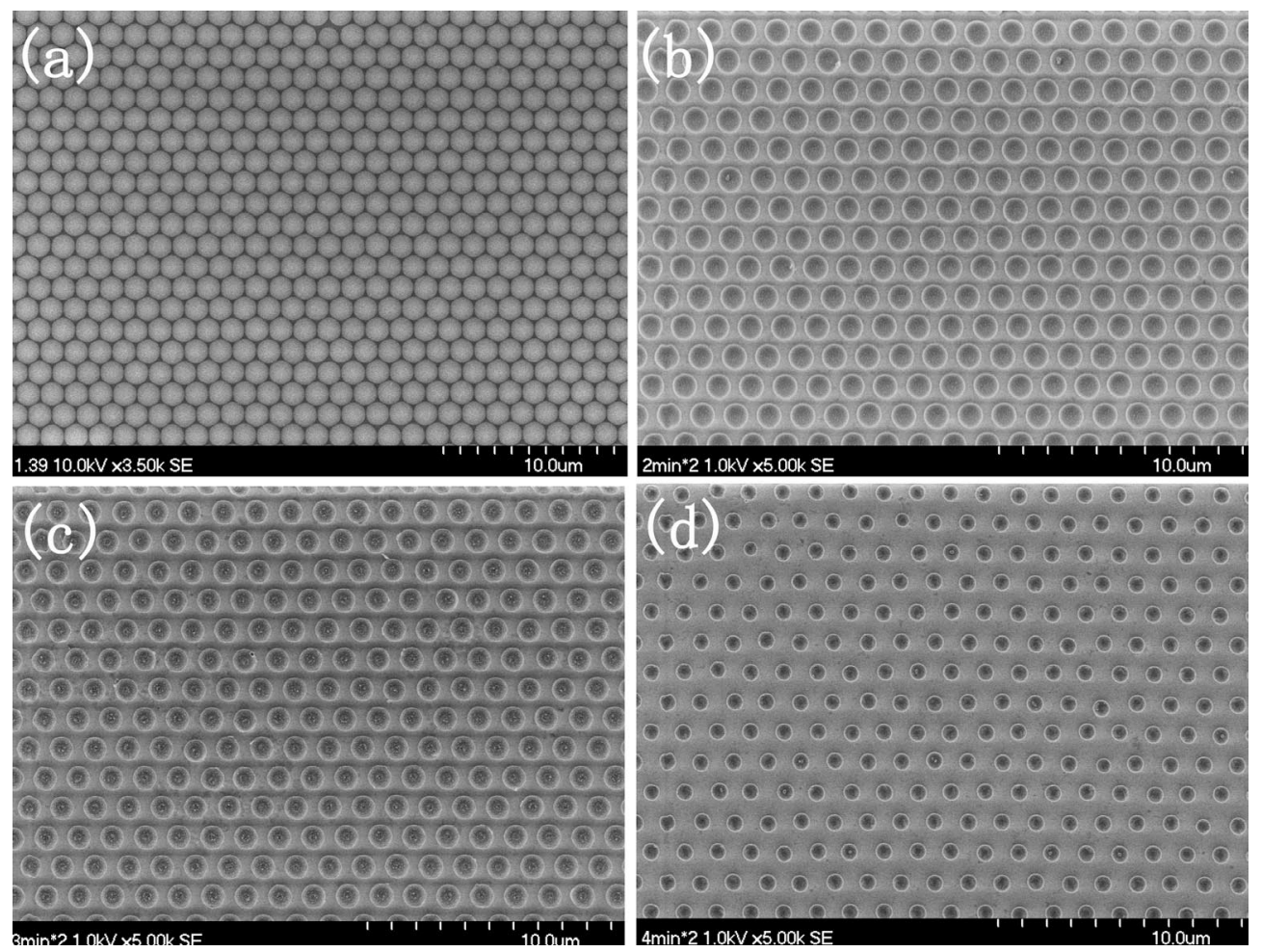

Figure S1. Figure caption. Reduction of the sizes of PS microspheres by oxygen 
plasma etching. SEM images of the original PS microspheres pattern (a) and the patterns after oxygen plasma etching for $4 \mathrm{~min}(\mathrm{~b}), 6 \mathrm{~min}(\mathrm{c})$, and $8 \mathrm{~min}(\mathrm{~d})$ at the same density of oxygen plasma $(\sim 500 \mathrm{~W})$.
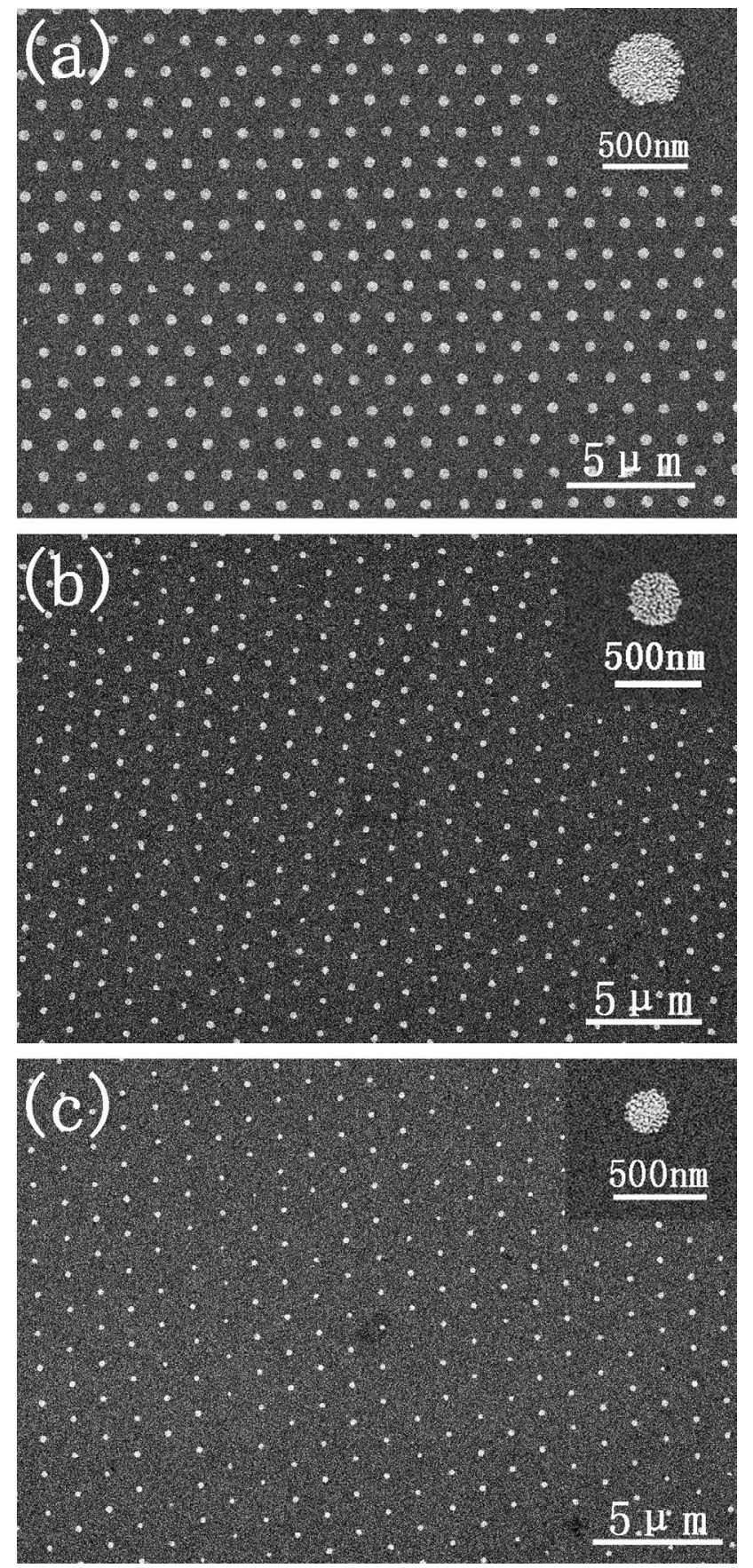

Figure S2. Figure caption. Adjustment of the size of the Au clusters by the duration of 
oxygen plasma etching while the heating time on the $110 \square$ hot plate was fixed at 2 min. SEM images of the Au particle patterns obtained after the samples in Figure S1(b)-S1(d) were removed of the PS microspheres by ultrasonication and etched with an aqueous $\mathrm{Fe}^{3+} /$ thiourea solution. The average size of the Au clusters was about 450nm, 320nm, and 250nm for (a), (b), and (c), respectively. Insets are magnified images of single particles.
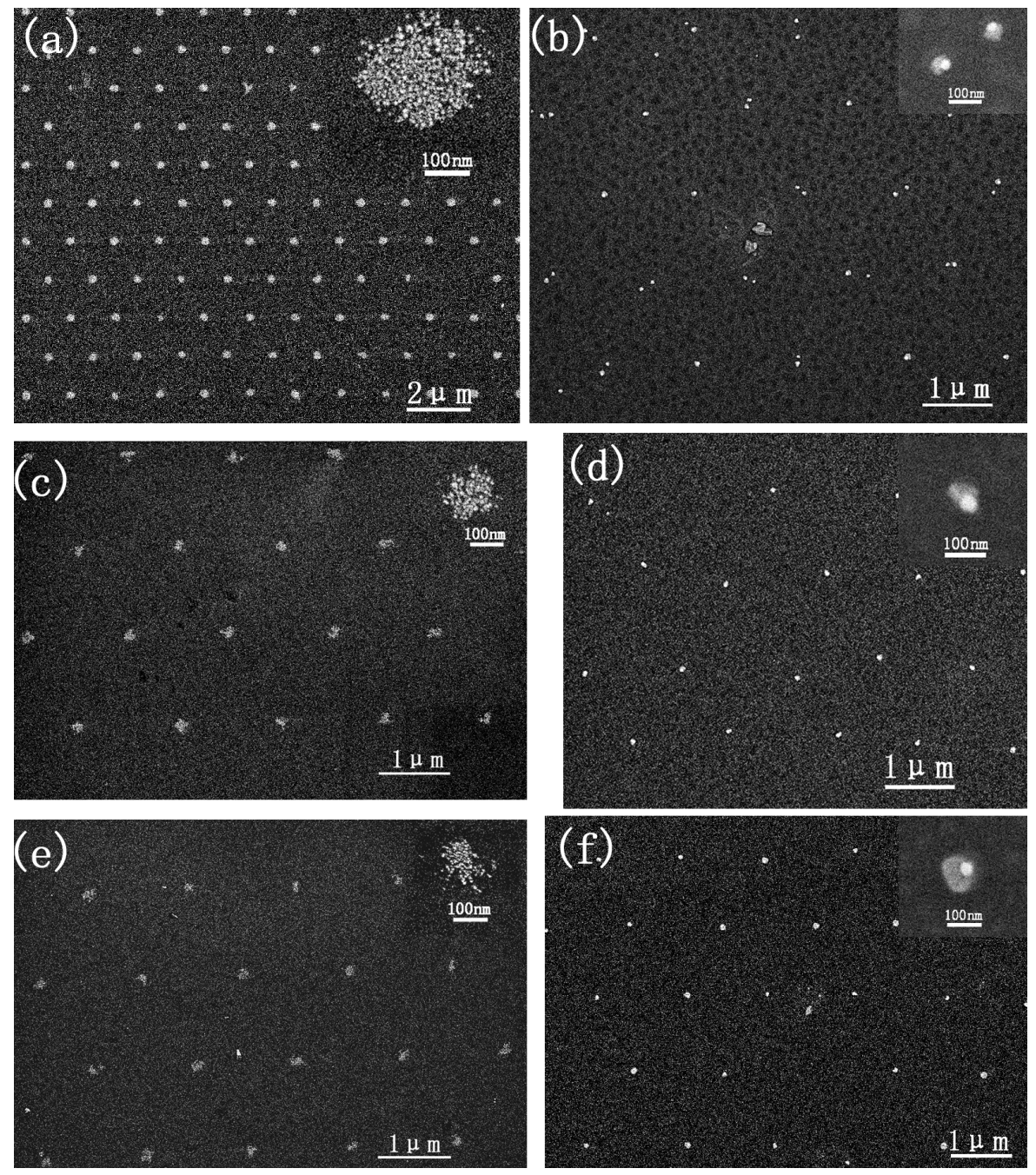

Figure S3. Figure caption. Morphology change of Au particle patterns before and after annealing for $10 \mathrm{~min}$ at $910 \square$. SEM images of Au particles with an original average 
diameter of about 300nm (a) before and (b) after annealing; 200nm (c) and (d); 150nm (e) and (f). Insets are the magnified images of individual Au particle sites. 